\title{
The Chemical Compositions of the Thoracic Aorta in Diabetics and Nondiabetics
}

\author{
Tokutaro Sato, Tsuyoshi Saito and Kaoru Yoshinaga \\ The Second Department of Internal Medicine, Tohoku \\ University School of Medicine, Sendai 980
}

\begin{abstract}
Sato, T., Saito, T. and Yoshinaga, K. The Chemical Compositions of the Thoracic Aorta in Diabetics and Nondiabetics. Tohoku J. exp. Med., 1985, 147 (4), 357-364 Chemical analysis of the composition of the thoracic aorta was performed in 19 age- and sex-matched pairs of diabetic and nondiabetic autopsy subjects. Contents of total lipid, cholesterol, triglyceride, phospholipid, free fatty acid and collagen in the aortic wall were greater in cases with advanced atherosclerosis than in those with less advanced atherosclerosis. According to severity of atherosclerosis, the ratio of cholesterol to total lipid in the aortic wall increased, while the ratios of triglyceride, phospholipid and free fatty acid to total lipid decreased. The cholesterol concentration in the aorta increased with dry weight of the aorta per area. The rate of increase was 2.6 times higher in diabetics than in nondiabetics. These results suggest that content of cholesterol in the aortic wall increases markedly according to the advance of atherosclerosis, especially in the diabetics. $\longrightarrow$ atherosclerosis ; diabetes ; lipid ; cholesterol ; collagen
\end{abstract}

Numerous reports have appeared regarding the chemical composition of human aorta in health and disease, and it has been shown that the chemical composition of the aorta, especially the content of cholesterol, total lipid, ash, collagen and mucopolysaccharides are related both to the severity of the atherosclerotic lesions and total area involved with atherosclerosis (Schönheimer 1926; Buck 1951; Buddecke 1958; Greer et al. 1958; Smith 1965a, b; Smith et al. 1967).

It is generally accepted that diabetes is associated with an increased incidence of atherosclerosis, which occurs at earlier age and in more advanced severity than nondiabetics. It was anticipated that the chemical composition of the aorta would reflect these findings. However, there are a few reports concerning the chemical composition of the diabetic aorta, and no specific feature has been demonstrated for atherosclerosis of diabetes (Bürger 1954; Bertelsen 1968; Brosnan et al. 1973). The present study, therefore, involves a comparison of the chemical composition of the aorta from diabetic and nondiabetic autopsy subjects. The tunica intima and the tunica media both grow in thickness by age and

Received September 4, 1984 ; accepted for publication September 25, 1985. 
atherosclerosis (Bertelsen 1968), and it is difficult to separate them in cases with advanced atherosclerosis. Therefore, both of the tunica intima and the tunica media were subjected together to chemical analysis in this study.

\section{Materials AND Methods}

Chemical analysis of the compositions of the thoracic aorta was performed in 19 ageand sex-matched pairs of diabetic and nondiabetic autopsy subjects, consisting of 16 men and 3 women. Main causes of death were cerebrovascular accident, renal failure, cancer and myocardial infarction. The average ages of the diabetics and nondiabetics were $62.7 \pm 10.9$ (S.D.) and $62.5 \pm 10.0$ (S.D.) years, respectively. Clinical data of diabetics are shown in Table 1. Duration of diabetes, recorded in 17 cases, ranged from 2 to 19 years with an average of 8.1 years. Treatment of diabetes was diet alone in 2 cases, oral hypoglycemic agent in 3 cases, insulin in 13 cases and unknowm in one case. Fasting blood glucose concentration, recorded in 16 diabetics, ranged from 90 to $728 \mathrm{mg} / 100 \mathrm{ml}$ with an average of $252 \mathrm{mg} / 100$ $\mathrm{ml}$. Glucosuria was detected in all the diabetics. In the nondiabetic group, diabetic predisposition and glucosuria were negative, and fasting blood glucose concentration was normal in all the subjects.

Macroscopically, ulceration of the intima was noted in the aorta from 6 diabetics and 6 nondiabetics, advanced plaque formation in the aorta from 12 diabetics and 7 nondiabetics.

The tunica media and the tunica intima of the formaldehyde-fixed aorta were rinsed with water and dried in air after measuring its surface area. The dried aorta was weighed, minced and ground by a coffee-mill and extracted in a mixture of chloroform and methanol (1:1) with occational shaking for 12 hours. The supernatant was removed after centrifugation at $3,000 \mathrm{rpm}$ for $20 \mathrm{~min}$. The same procedure was repeated 13 more times. The extract was dried under air stream, and weighed to determine total lipid content in the aorta. Total lipid was dissolved in $10 \mathrm{ml}$ of a mixture of chloroform-methanol $(1: 1)$ and the cholesterol concentration in the extract was determined by the Zack's method (1954), phospholipid by the Bartlett's method (1959), free fatty acid by the Itaya's method (1965 Itaya and Ui), triglyceride by Trigly-kit of Eiken Chem. Co., Ltd. The extracted residue of the aorta was dried in air, and a part of the extracted residue was hydrolyzed in $6 \mathrm{~N}$ constant boiling $\mathrm{HCl}$ under nitrogen at $105^{\circ} \mathrm{C}$ for $20 \mathrm{hr}$. Hydroxyproline concentration in the hydrolysate of the extracted residue was determined by the Kivirikko's method (Kivirikko et al. 1967). The contents of collagen was calculated by multiplying the hydroxyproline content by 7.7 (Newamn and Logan 1950).

\section{RESUlts}

As shown in Table 2, dry weight and content of total lipid per area of the aorta apperaed to increase according to the advance of atherosclerosis, and content of total lipid was especially greater in the ulcerated aorta of diabetics. Contents of cholesterol, triglyceride, phospholipid and free fatty acid in the aortic wall were also greater in cases with advanced atherosclerosis of both diabetics and nondiabetics, especially the ulcerated aorta of diabetics. The ratio of contstituent lipids to total lipid in the aorta is also shown in Table 2. According to the advance of atherosclerosis, the proportion of cholesterol increased, although the proportion of all the other lipids such as triglyceride, phospholipid and free fatty acid decreased.

The content of collagen in the extracted residue of the aorta increased 
TABLE 1. Clinical picture of the diabeties

\begin{tabular}{|c|c|c|c|c|c|c|c|}
\hline Case & $\begin{array}{c}\text { Age } \\
\text { (years) }\end{array}$ & Sex & $\begin{array}{l}\text { Family } \\
\text { history } \\
\text { of } \\
\text { diabetes }\end{array}$ & $\begin{array}{c}\text { Duration } \\
\text { of } \\
\text { diabetes } \\
\text { (years) }\end{array}$ & $\begin{array}{c}\text { Treatment } \\
\text { of } \\
\text { diabetes }\end{array}$ & Glucosuria & $\begin{array}{c}\text { Fasting } \\
\text { blood } \\
\text { glucose } \\
(\mathrm{mg} / 100 \mathrm{ml})\end{array}$ \\
\hline GN & 49 & $\mathrm{M}$ & $(-)$ & & Insulin & $(+)$ & 146 \\
\hline $\mathrm{KT}$ & 85 & $\mathrm{~F}$ & $(-)$ & 19 & & $(\mathrm{H})$ & \\
\hline SY & 69 & M & & 7 & Insulin & $($ \#) & 728 \\
\hline MT & 53 & $\mathrm{~F}$ & $(-)$ & 10 & Insulin & $(\#)$ & 227 \\
\hline $\mathrm{SN}$ & 36 & M & $(-)$ & 17 & Insulin & $($ m) & \\
\hline OT & 53 & M & $(+)$ & 6 & Diet & $(+)$ & 210 \\
\hline SS & 69 & M & & 3 & Insulin & $(H)$ & 174 \\
\hline $\mathrm{KM}$ & 66 & M & $(+)$ & 3 & Insulin & $(+)$ & 147 \\
\hline SY & 54 & M & $(-)$ & 2 & Insulin & ( \#) & 98 \\
\hline SK & 72 & M & $(-)$ & 12 & Oral agent & ( \#) & 225 \\
\hline HS & 61 & M & $(-)$ & & Diet & ( \#) & 335 \\
\hline KK & 63 & M & $(-)$ & 12 & Insulin & (H) & \\
\hline TK & 69 & M & $(-)$ & 3 & Insulin & $(+)$ & 90 \\
\hline $\mathrm{AH}$ & 69 & M & $(-)$ & 2 & Oral agent & $(H)$ & 315 \\
\hline SS & 56 & $\mathrm{~F}$ & $(-)$ & 7 & Insulin & $($ \#) & 267 \\
\hline HJ & 73 & $\mathrm{M}$ & & 10 & Insulin & $(+)$ & 233 \\
\hline ST & 64 & $\mathrm{M}$ & $(+)$ & 12 & Insulin & $($ \#) & 325 \\
\hline SS & 61 & $\mathrm{M}$ & $(-)$ & 3 & Insulin & $(+)$ & 403 \\
\hline $\mathrm{OK}$ & 70 & $\mathrm{M}$ & & 10 & Oral agent & $(+)$ & 114 \\
\hline
\end{tabular}

according to the advance of atherosclerosis in both of diabetics and nondiabetics.

Similar results were obtained when dry weight of the aorta per area was used as an index of atherosclerosis (Table 3). The correlation coefficient between dry weight of the aorta and content of each of total lipid, cholesterol, triglyceride, phosholipid, free fatty acid and collagen in diabetics were $+0.774(p<0.001)$, + $0.807(p<0.001),+0.685(p<0.001),+0.784 \quad(p<0.001),+0.788 \quad(p<0.001)$, and $+0.836(p<0.001)$, respectively. The values in nondiabetics were +0.506 $(p<0.05),+0.516(p<0.05),+0.340(p<0.05),+0.172(p>0.05),+0.449(p<$ $0.05)$, and $+0.807(p<0.001)$. The relation between the increase in the cholesterol concentration in the aorta and the dry weight of the aorta per area was represented by the regression equations; $\mathrm{y}=-6.05+0.14 \mathrm{x}$ for diabetics, and $y=-1.80+0.053 \mathrm{x}$ for nondiabetics (Fig. 1). The slope of the regression line for diabetics was 2.6 times larger than the for nondiabetics, and a statistically significant difference was noted between the slope of the two lines.

The correlation coefficient between ratio of constituent to total lipid in the aorta and dry weight of the aorta per area showed positive value for total cholesterol and negative values for the other lipid compositions. Statistically significant difference was detected in the diabetic group. 
TABLE 2. Dry weight and chemical compositions of the aorta

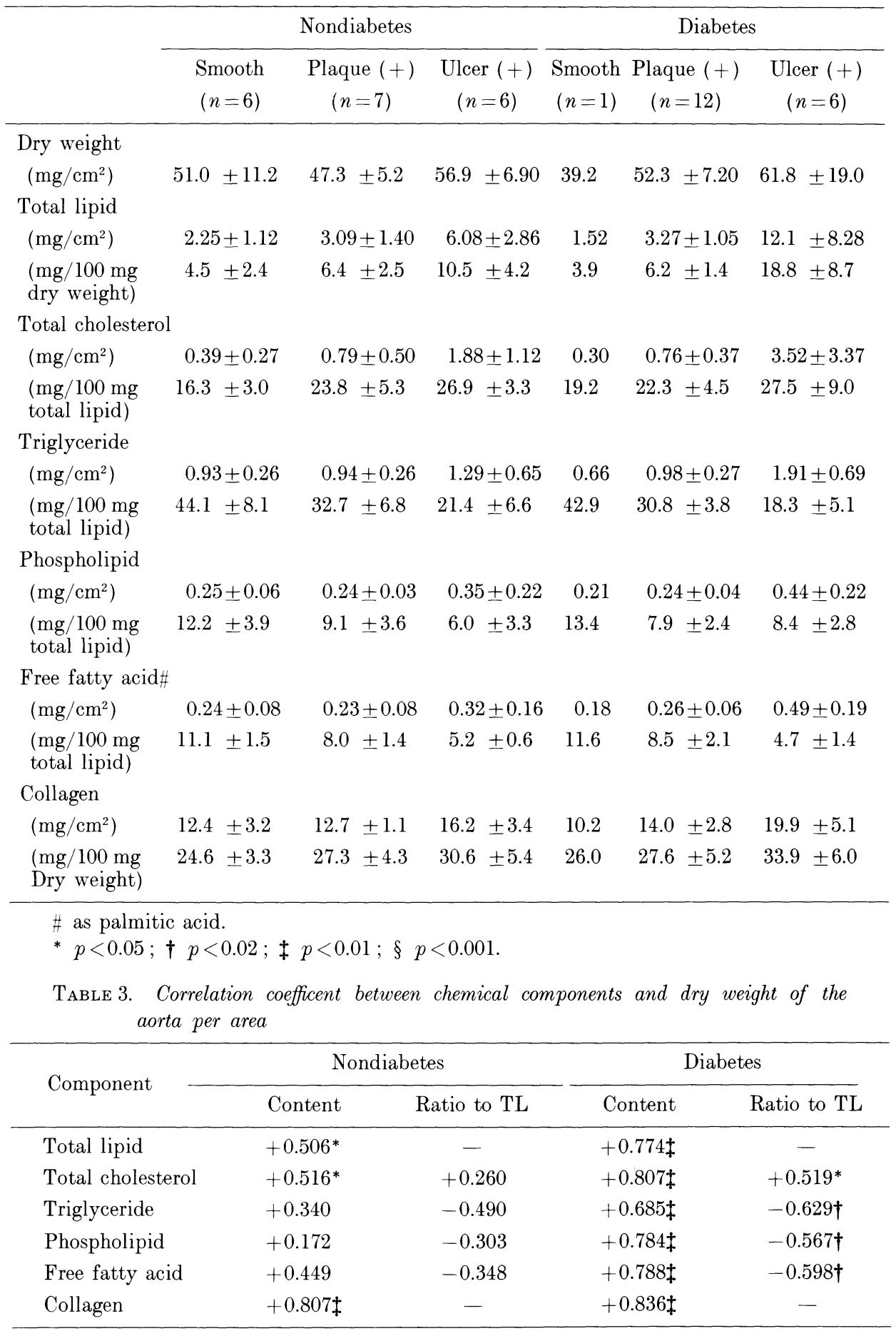

TL : total lipid. ${ }^{*} p<0.05, \dagger p<0.01, \ddagger p<0.001$. 


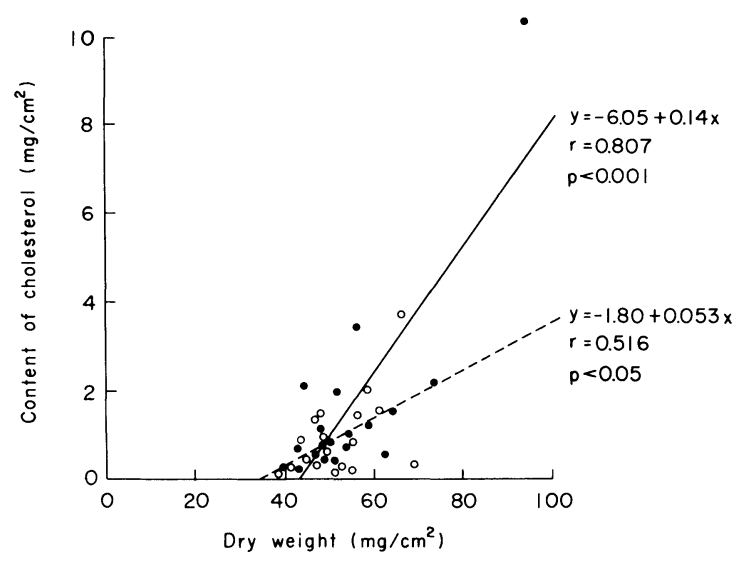

Fig. 1. Relation between content of cholesterol and dry weight of the aorta per area.

diabetes $(\bullet,-)$, nondiabetes $(\circ, \cdots \cdots)$.

\section{Discussion}

Chemical analysis of lipids, collagen and minerals in the human aorta in health and disease was already performed (Schönheimer 1926; Buck 1951; Buddecke 1958; Greer et al. 1958; Smith 1965a; Smith et al. 1967), however, a few reports are available to date on studies of the chemical composition of the diabetic aorta (Bürger 1954; Bertelsen 1968; Brosnan et al. 1973). In this study, chemical analysis was performed to see changes in the composition of the diabetic aorta. The chemical components studied involved total lipid, cholesterol, triglyceride, phospholipid, free fatty acid and collagen.

In the present study, chemical determination was performed on samples already fixed in formaledehyde. Mendez and Tejada (1969) reported that recovery of lipid from the aorta was satisfactory even after fixation and staining. In this study, each of three thoracic aorat was divided into 2 groups; one was kept frozen untill use and the other was fixed in $10 \%$ formaldehyde for 6 months. The aorta of the both groups was extracted in a mixture of chloroform : methanol (1: 1). Total cholesterol concentration in the extract was determined. The value for the formaldehyde-fixed aorta was $93.8 \%$ of that for the aorta without fixation. Further, values obtained in the present study are similar to those reported in the previous determinations on the aorta without fixation in formaldehyde (Meeker and Jobling 1934; Buck and Rossiter 1951; Sata et al. 1973), except phospholipid which showed lower values.

Chemically, the collagen molecule is characterized first of all by its content of hydroxyproline which constitutes about 13\% (Newman and Logan 1950). In the present study, quantitative collagen determination was performed by hydroxyproline analysis, and the value of $24.6 \%$ for the grossly normal aorta of non- 
diabetics agrees with the results by Buddecke (1958).

According to the results by Mendez and Tejada (1969), single visual evaluation of atherosclerosis gives a good result as does the system utilizing specific staining procedure, and dry weight of the aorta is also a good index of severity of atherosclerosis. In the present study, degree of atherosclerosis of the aorta was divided into three groups according to the appearence (i.e., grossly normal, plaque-formed, and ulcerated). Dry weight of the aorta per area was also chosen as an index of the severity of atherosclerosis.

Böttcher and Vangent (1961), Whitehead et al. (1962), Smith (1965a), Mizuguchi (1968), and Mead and Gauge (1961), reported that total lipid increased progressively with advancing atherosclerotic process, and that the proportion of triglyceride and phospholipid decreased in nondiabetics. The results obtained in the present study are similar to those reported by them, showing increased proportion of cholesterol and decreased proportions of other lipids such as triglyceride, phospholipid and free fatty acid to total lipid with progress of sclerosis of the aorta. Similar results were obtained by comparison between lipid content and dry weight of the aorta per area; content of total lipid and cholesterol increased markedly by dry weight of the aorta, while those of other lipid components as triglyceride, phospholipid and free fatty acid decreased.

Earlier studies proved that esterified cholesterol increased according to the advance of atherosclerosis and with an age (Smith 1965a, b; Proudlock et al. 1973). In the present study, the average ratio of esterified cholesterol to total cholesterol increased slightly with severity of atherosclerosis in nondiabetics; $67.7 \%$ in the grossly normal aorta, $76.5 \%$ in the plaque-formed aorta and $79.1 \%$ in the ulcerated aorta. In diabetics, the ratio was $76.6 \%$ in the plaque-formed aorta and $65.0 \%$ in the ulcerated aorta.

As well as lipid and mucopolysaccharides, collagen content has been shown to increase in the atherosclerotic aorta (Smith 1965b; Kanabrocki et al. 1960; Bertelsen 1968). The results in the present study, also showed that content of collagen was higher in the defatted intima and media from ulcerated aorta than plaque-formed aorta.

By comparative analysis of the aortic wall from diabetics and nondiabetics, Bertelsen demonstrated that no significant difference was demonstrated in the content of lipid and collagen (1968). Brosnan et al. (1973) demonstrated that the contents of collagen in the canine aorta is not influenced by insulin deficiency or by insulin replacement therapy. On the other hand, Bürger (1954) reported that contents of total lipid and cholesterol in the aorta were higher in the diabetics than in the nondiabetics. The difference among these studies may depend on the difference of severity of atherosclerosis between diabetics and nondiabetics.

In the present study, the total lipid and cholesterol concentration in the aorta increased by dry weight of the aorta per area in both diabetics and nondiabetics. The rate of increase in diabetics was 2.6 times higher that in nondiabetics, and 
significant difference between the both groups was noted in the cholesterol concentration. This result suggests that the increase of cholesterol in the atherosclerotic aorta is more pronounced in diabetes than in nondiabetes.

\section{References}

1) Bartlett, G.R. (1959) Phosphorus assay in colum chromatography. J. biol. Chem., 234, 466-468.

2) Bertelsen, S. (1968) Chemical studies on the arterial wall in relation to atherosclerosis. Ann N.Y. Acad. Sci., 149, 643-654.

3) Böttcher, C.J.F. \& Vangent, C.M. (1961) Changes in the composition of phospholipid fatty acids associated with atherosclerosis in the human aortic wall. $J$. Atheroscler. Res., 1, 36-46.

4) Brosnan, M.E., Sirek, O,V., Sirek, A. \& Prezybylska, K. (1973) Effect of pancreatectomy, with and without hypophysectomy, and of insulin treatment on the composition of canine aorta. Diabetes, 22, 397-402.

5) Buck, R.C. (1951) Minerals of normal and atherosclerotic aorta. Arch. Path., 51, $319-328$.

6) Buck, R.C. \& Rossiter, R.J. (1951) Lipids of normal and atherosclerotic aorta. Arch. Path., 51, 224-237.

7) Buddecke, E. (1958) Untersuchungen zur Chimie der Arterienwand. II Arteriosklerotishe Veranderungen am Aortenbindegewebe des Menschen. Z. physiol. Chim., 310, 182-198.

8) Bürger, M. (1954) Die angiochemischen Veränderungen an den grossen Gefässen der Diabetiker. In : Angiopathia Diabetica, edited by M. Bürger, Georg Thieme Verlag, Stuttgart pp. 8-14.

9) Greer, J.C., Strong, J.P., McGill, H.C, Nynian, M.A. \& Werthessen, N.T. (1958) Correlation of intimal sudanophilia and cholesterol analysis of three layers of human aorta. Proc. Soc. exp. Biol. Med., 98, 260-263.

10) Itay, K. \& Ui, M. (1965) Colorimetric determination of free fatty acids in biological fluids. J. Lipid Res., 6, 16-20.

11) Kanabrocki, E.L., Tels, L.G. \& Kaplan, E. (1960) Calcium, cholesterol, and collagen levels in human aortas. J. Geront., 15, 383-387.

12) Kivirikko, K.I., Latinen, O. \& Prockop, D.J. (1967) Modification of a specific assay for hydroxyproline in urine. Anal. Biochem., 19, 249-255.

13) Mead, J.I. \& Gouze, M.L. (1961) Alterations in aorta lipids with advancing atherosclerosis. Proc. Soc. exp. Biol. Med., 106, 4-7.

14) Meeker, D.R. \& Jobling, J.W. (1934) A chemical study of atherosclerotic lesions in human aorta. Arch. Path., 18, 252-306.

15) Mendez, J. \& Tejada, C. (1969) Relation of severity of atherosclerosis to chemical composition of human aorta. Amer. J. clin. Path., 51, 113-118.

16) Mizuguchi, T. (1968) Study on lipids and fatty acids of the plasma and aortic walls in human and experimental atherosclerosis. Jap. Heart $J$., 9, 34-45.

17) Newman, R.E. \& Logan, M.A. (1950) The determination of hydroxyproline. $J$. biol. Chem., 184, 299-306.

18) Proudlock, J.W., Day, A.J. \& Tume, P.K. (1973) Cholesterol-esterifing enzymes of foam cells isolated from atheerosclerotic rabbit intima. Atherosclerosis, 18, 451-458.

19) Sata, T., Shigemi, U., Murakami, H., Okabayashi, T. \& Nakamura, M. (1973) Studies on lipids of coronary artery and aorta. Jap. Circulat. J., 37, 261-267.

20) Schönheimer, R. (1926) Zur der gedunsenden und der atherosklerotishen Aorta. Hoppe-Seyler's Z. physiol. Chim., 160, 61-76.

21) Smith, E.B. (1965a) The infuence of age and atherosclerosis on the chemistry of 
aortic intima. Part 1. The lipid. J. Atheroscler. Res., 5, 224-240.

22) Smith, E.B. (1965b) The influence of age and atherosclerosis on the chemistry of aortic intima. Part 2. Collagen and mucopolysaccharides. J. Atheroscler. Res., 5, $241-248$.

23) Smith, E.B., Evans, P.H. \& Downham, M.D. (1967) Lipid in the aortic intima, the correlations of morphological and chemical characteristics. J. Atheroscler. Res., 7, 171-186.

24) Whitehead, T.P., Alexander, M.K., Barrowcliff, D.F., Prior, A.P. \& Marsh, N. (1962) Lipid and mineral matter in coronary arteries and aorta. J. Atheroscler. Res., 2, 199209.

25) Zack, B., Dickerman, R.C., White, E.G., Burnett, H. \& Cherney, P.J. (1954) Rapid estimation of free and total cholesterol. Amer J. clin. Path., 24, 1307-1315. 